\title{
Bruxismo na infância: fatores etiológicos e possíveis fatores de risco
}

\section{Bruxism in children: etiologic factors and possible risk factors}

\author{
Luana Cardoso Cabral ${ }^{1}$ \\ ANA JÚLIA DA COSTA LOPES ${ }^{2}$ \\ Marcos Boaventura de Moura ${ }^{1}$ \\ RaISSA RAMOS DA SILVA ${ }^{3}$ \\ Alfredo Júlio Fernandes Neto 4 \\ Paulo Cézar Simamoto JúNIOR ${ }^{4}$
}

\begin{abstract}
RESUMO
Introdução: O bruxismo é considerado um hábito parafuncional caracterizado pelo apertamento e/ou ranger dos dentes de maneira inconsciente e estereotipada. Considerando que a prevalência desse hábito é maior durante a infância, havendo uma diminuição com o passar da idade, o bruxismo infantil tornou-se preocupante devido ao seu impacto negativo na qualidade de vida da criança e de seus familiares.

Objetivo: Este estudo teve como objetivo revisar a literatura quanto à etiologia e fatores de risco associados a essa atividade parafuncional.

Metodologia: Foram incluídos estudos clínicos com pacientes pediátricos diagnosticados com bruxismo e que abordavam fatores de risco ou etiológicos dessa parafunção, e excluídos revisões de literatura, casos clínicos, opiniões pessoais, trabalhos não publicados ou aqueles que cuidavam apenas do tratamento do bruxismo infantil. Buscas avançadas foram conduzidas nas bases de dados Pubmed, Cochrane, LILACS e Medline, e a seleção dos artigos foi realizada, independentemente, por dois revisores. A qualidade metodológica dos artigos selecionados foi avaliada por meio da ferramenta Qu-ATEBS. Foram identificados 146 artigos ao total em pesquisa eletrônica, após leitura de título e resumo, e avaliação em texto completo, apenas seis artigos foram incluídos em síntese qualitativa. Entre os artigos incluídos, os autores observaram que vários fatores de risco estavam relacionados ao bruxismo, como refluxo gastrointestinal, dor de estômago, crises respiratórias, enurese noturna, onicofagia, sialorreia noturna, dificuldade para dormir, entre outros. Já a etiologia é multifatorial, podendo envolver fatores psicológicos, sistêmicos, genéticos e locais.

Conclusão: É de extrema importância que o cirurgião dentista tenha conhecimento tanto dos fatores de risco quanto da etiologia do bruxismo, para o desenvolvimento de uma melhor abordagem preventiva e intervenções efetivas.
\end{abstract}

Palavras-chave: Bruxismo. Bruxismo do sono. Criança.

\begin{abstract}
Introduction: Bruxism is a parafunctional habit characterized by the involuntary grinding and/or clenching of the teeth. The prevalence is highest during childhood and adolescence and there is a decline with increasing age. Because of this prevalence, the bruxism in children has become worrisome due to the negative impact on children's quality of life and their families.

Objective: The aim of this study was to review the literature regarding the etiology and risk factors associated with childhood bruxism.
\end{abstract}

\footnotetext{
${ }^{1}$ Cirurgião-dentista. Especialista em Prótese Dentária. Mestre e Doutor em Odontologia (Reabilitação Oral). Universidade FEDERAL de UberLÂNDIA, UBERLÂNDIA, MG, Brasil.

${ }^{2}$ Cirurgiã-dentista. Mestranda em Odontologia (Reabilitação Oral). Universidade Federal de Uberlândia, Uberlândia, MG, BRASIL.

${ }^{3}$ Graduação em odontologia. Universidade Federal de Uberlândia, Uberlândia, MG, Brasil.

${ }^{4}$ Cirurgião-dentista. Especialista em Prótese Dentária. Mestre e Doutor em Odontologia (Reabilitação Oral). Professor das disciplinas de Prótese fixa, Oclusão e Materiais Dentários. Universidade Federal de Uberlândia, Uberlândia, MG, Brasil
} 
Methodology: The descriptors bruxism, sleep bruxism, child and etiology were used for electronic search in the Pubmed, Cochrane, LILACS, Medline, Scielo and Scientific Journal. 146 articles were found in the first Advanced Search, after passing through the inclusion and exclusion criteria there were only 06. Among the selected articles, the authors note that several factors were related to bruxism, including gastrointestinal reflux, stomach pain, respiratory crises, enuresis night, nail biting, nocturnal drooling, trouble sleeping, among others. The etiology of bruxism is multifactorial, involving psychological factors, systemic, genetic, occupational and local.

Conclusion: It is extremely important that the dentist has the knowledge of the risk factors involved with bruxism to better preventive approach, aid in the diagnosis and effective interventions.

Key-words: Bruxism. Sleep bruxism. Child.

\section{INTRODUÇÃO}

O bruxismo é um hábito oral parafuncional caracterizado pelo ato involuntário de apertar e/ou ranger dos dentes de modo repetitivo e estereotipado, podendo ocorrer em vigília ou durante o sono. ${ }^{1,2} \mathrm{~A}$ ampla variedade de métodos de diagnóstico desse distúrbio dificulta a análise dos dados de sua prevalência, porém se sabe que em crianças e adolescentes a prevalência é maior quando comparada em adultos, com índice de 17\% e $8 \%$ respectivamente, não apresentando diferenças significativas entre gêneros. ${ }^{3,4,5}$

Considerando a prevalência, o bruxismo infantil tornou-se preocupação nos últimos anos em decorrência do seu impacto negativo na qualidade de vida da criança e de seus familiares. ${ }^{6}$ Tal desordem pode ocasionar sequelas significativas ao sistema estomatognático, como desgastes dentários, desordens temporomandibulares, dores de cabeça ou fadiga dos músculos mastigatórios, além de complicações sociais como comprometimento da qualidade do sono. Crianças com bruxismo tendem a dormir menos de oito horas por dia, e a privação do sono reflete diretamente em seu desempenho educacional. . $^{7,89}$

A etiologia do bruxismo é multifatorial e pode ser de origem psicológica, sistêmica ou genética, havendo influência potencial do sistema nervoso central. ${ }^{5,10}$ Sua etiologia psicológica está relacionada com a presença de ansiedade, hiperatividade e estresse ligados às tarefas do cotidiano, além de traços de personalidade. ${ }^{11}$ Os fatores sistêmicos incluem alterações como a rinite, sinusite, asma e outras complicações respiratórias alérgicas. ${ }^{12}$ $O$ fator genético diz respeito à herança genética e sua contribuição substancial no fenótipo do bruxismo. $^{2}$

Um consenso internacional realizado por especialistas ${ }^{2}$ culminou na criação de um sistema de diagnóstico em escala para o bruxismo, no qual o diagnóstico definitivo deve ser ancorado na associação entre relato pessoal, detecção de sinais e sintomas por meio da avaliação clínica, e achados polissonográficos. A polissonografia do sono permite o registro quantitativo dos episódios da atividade muscular mastigatória rítmica (AMMR), sendorecomendadapara confirmação ou complementação do diagnóstico. ${ }^{2,13}$

Não há cura para o bruxismo, tampouco tratamento específico para a patologia infantil. ${ }^{14,15} \mathrm{O}$ acompanhamento multiprofissional envolvendo odontopediatras, psicólogos, pediatras e otorrinolaringologistas é de fundamental importância. $O$ tratamento paliativo envolve o uso de fármacos, placas oclusais, acompanhamento psicológico e controle dos fatores de risco. ${ }^{9}$

Considerando a elevada prevalência do bruxismo infantil na prática odontológica diária e suas graves consequências ao aparelho estomatognático e a qualidade de sono do indivíduo, o conhecimento acerca dos possíveis fatores de risco e fatores etiológicos é de fundamental importância para se estabelecer uma abordagem preventiva e terapêutica 
individualizada e mais efetiva. Nesse contexto, o objetivo deste trabalho foi revisar a literatura quanto aos diferentes fatores etiológicos e fatores de risco associados ao bruxismo infantil.

\section{Materiais e mÉtodos}

\section{Critérios de Elegibilidade}

Foram selecionados estudos clínicos com pacientes pediátricos diagnosticados com bruxismo e que abordavam os fatores etiológicos ou os fatores de risco dessa parafunção. Revisões de literatura, casos clínicos, opiniões pessoais, trabalhos não publicados ou aqueles que cuidavam apenas do tratamento do bruxismo infantil foram excluídos.

\section{Seleção dos Estudos}

Buscas avançadas foram conduzidas nas bases de dados PubMed, Cochrane, LILACS e Medline, por meio de descritores obtidos no dicionário DeCs/Mesh, foram utilizados nas buscas os seguintes descritores: etiology, child, sleep bruxism, bruxism. Foi realizada uma busca manual e a literatura cinza acessada por meio do Google acadêmico.

A seleção dos artigos foi realizada em duas etapas. $\mathrm{Na}$ primeira etapa, o título e o resumo dos estudos foram avaliados de modo independente por dois revisores. Estudos que não preenchiam o critério de inclusão foram descartados. Na etapa 2, os artigos foram avaliados em texto completo, independentes, pelos mesmos dois revisores. Os desacordos em qualquer uma das etapas foram resolvidos pelo terceiro observador. Dois autores coletaram de modo independente as características metodológicas e informações necessárias dos artigos incluídos. Qualquer desacordo entre os autores foi resolvido por meio de discussão e consenso mútuo.

A qualidade metodológica dos artigos selecionados foi avaliada por meio da ferramenta de avaliação de qualidade de estudos experimentais de bruxismo (QuATEBS $)^{16}$ com o objetivo de calcular o risco de viés. Sete itens dispostos como perguntas eram avaliados em uma escala de cinco valores. A pontuação máxima é de 70 pontos, uma pontuação de 0 a 50 pontos define o artigo como de baixa qualidade e uma pontuação de 51 a 70 pontos o define como de alta qualidade. Os dois revisores pontuaram cada item, de maneira independente, com valores de 1 a 5 . Os desacordos entre os dois revisores foram resolvidos pelo terceiro revisor.

\section{RESULTADOS}

Foram identificados um total de 146 artigos na busca bibliográfica, sendo que 140 artigos foram excluídos por não se adequarem aos critérios de inclusão e exclusão, os demais seis artigos foram incluídos em síntese qualitativa.

Após avaliação da metodologia dos artigos por meio do Qu-ATEBS, dois artigos foram classificados como de baixa qualidade metodológica e os demais como de alta qualidade (Tabela 1).

\section{Síntese dos artigos incluídos}

De um total de 146 artigos, seis preencheram os critérios de inclusão deste estudo e foram classificados quanto ao risco de viés. As características metodológicas e informações necessárias dos artigos incluídos estão presentes na tabela. 
Tabela 1. Avaliação metodológica da qualidade dos artigos para estudo experimental do bruxismo por meio da ferramenta Qu-ATEBS.

\begin{tabular}{|c|c|c|c|c|c|c|}
\hline $\begin{array}{l}\text { Ferramenta de avaliação de qualidade } \\
\text { para o estudo experimental do } \\
\text { bruxismo (Qu-ATEBS) }\end{array}$ & $\begin{array}{l}\text { Miller et } \\
\text { al. } \mathbf{2 0 0 3}^{17}\end{array}$ & $\begin{array}{l}\text { Simões-Zenari } \\
\text { e Bitar. } 2010^{6}\end{array}$ & $\begin{array}{l}\text { Miamoto et } \\
\text { al. 2011 }\end{array}$ & $\begin{array}{l}\text { Montaldo et } \\
\text { al. 2012 }\end{array}$ & $\begin{array}{l}\text { Ferreira- } \\
\text { Bacci Ado et } \\
\text { al. } 2012^{20}\end{array}$ & $\begin{array}{l}\text { Sakaguchi } \\
\text { et al. } \\
2014^{21}\end{array}$ \\
\hline $\begin{array}{c}\text { 1- Qualidade dos relatórios: Os obje- } \\
\text { tivos ou hipóteses dos estudos foram } \\
\text { claramente descritos? }\end{array}$ & 4 & 5 & 5 & 5 & 5 & 5 \\
\hline $\begin{array}{l}\text { Qualidade do design: Os objetivos ou } \\
\text { hipóteses foram ancorados em teoria } \\
\text { relevante? }\end{array}$ & 3 & 4 & 4 & 3 & 5 & 5 \\
\hline $\begin{array}{l}2 \text { - Qualidade do relatório: Os critérios } \\
\text { de elegibilidade, usados para selecio- } \\
\text { nar os participantes, foram suficiente- } \\
\text { mente descritos? }\end{array}$ & 4 & 3 & 5 & 3 & 3 & 3 \\
\hline $\begin{array}{l}\text { Qualidade do projeto: Os critérios de } \\
\text { elegibilidade foram adequados aos } \\
\text { objetivos deste estudo? }\end{array}$ & 3 & 3 & 4 & 3 & 3 & 3 \\
\hline $\begin{array}{l}3 \text { - Qualidade do relatório: Foi clara- } \\
\text { mente descrito se um grupo controle, } \\
\text { condição do grupo controle, ou uma } \\
\text { condição experimental foram utilizados? }\end{array}$ & - & 4 & 5 & 3 & - & 4 \\
\hline $\begin{array}{l}\text { Qualidade do projeto: O grupo contro- } \\
\text { le, a condição do grupo controle ou a } \\
\text { condição experimental foram apropria- } \\
\text { dos para este estudo? }\end{array}$ & - & 4 & 4 & 3 & - & 4 \\
\hline $\begin{array}{l}\text { 4- Qualidade do relatório: o design do } \\
\text { estudo foi descrito com suficiente deta- } \\
\text { Ihe para permitir a replicação? }\end{array}$ & 4 & 5 & 4 & 4 & 4 & 4 \\
\hline $\begin{array}{l}\text { Qualidade do projeto: O projeto do es- } \\
\text { tudo foi selecionado adequadamente } \\
\text { para os objetivos deste estudo? }\end{array}$ & 3 & 5 & 4 & 4 & 4 & 4 \\
\hline $\begin{array}{l}5 \text { - Qualidade do relatório: A tarefa } \\
\text { experimental de bruxismo foi descrita } \\
\text { com tal detalhe que a replicação seja } \\
\text { possível? }\end{array}$ & 4 & 5 & 5 & 4 & 5 & 4 \\
\hline $\begin{array}{l}\text { Qualidade do projeto: A tarefa experi- } \\
\text { mental de bruxismo foi apropriadamen- } \\
\text { te selecionada para os objetivos deste } \\
\text { estudo? }\end{array}$ & 5 & 5 & 5 & 4 & 4 & 4 \\
\hline $\begin{array}{c}\text { 6- Qualidade do relatório: Os métodos } \\
\text { e dados estatísticos foram suficiente- } \\
\text { mente descritos? }\end{array}$ & 5 & 4 & 5 & 5 & 2 & 5 \\
\hline $\begin{array}{l}\text { Qualidade do projeto: Os métodos e } \\
\text { dados estatísticos foram apropriados } \\
\text { para os objetivos deste estudo? }\end{array}$ & 5 & 4 & 5 & 5 & 2 & 5 \\
\hline $\begin{array}{l}\text { 7- Qualidade do relatório: As conclu- } \\
\text { sões do estudo foram adequadamente } \\
\text { formuladas? }\end{array}$ & 5 & 4 & 4 & 4 & 4 & 4 \\
\hline $\begin{array}{c}\text { Qualidade do design: Os objetivos e } \\
\text { hipóteses foram claramente abordados } \\
\text { nas conclusões e relevantes para os } \\
\text { objetivos? }\end{array}$ & 4 & 3 & 4 & 3 & 4 & 4 \\
\hline TOTAL & 49 & 58 & 63 & 53 & 45 & 58 \\
\hline
\end{tabular}


Tabela 2. Características metodológicas e informações necessárias dos artigos incluídos.

\begin{tabular}{|c|c|c|c|c|c|}
\hline Ano & Autores & Tipo de Estudo & $\begin{array}{c}\text { Tamanho da } \\
\text { amostra }\end{array}$ & Diagnóstico & $\begin{array}{l}\text { Fatores etiológicos } \\
\text { e Fatores de risco }\end{array}$ \\
\hline 2003 & $\begin{array}{l}\text { Miller, VA et al. } \\
2003^{17}\end{array}$ & Transversal & 118 & $\begin{array}{l}\text { Questionário de Hábitos de } \\
\text { Sono das Crianças (CSHQ). }\end{array}$ & Presença de enxaqueca. \\
\hline 2010 & $\begin{array}{l}\text { Simões-Zenari et } \\
\text { al. } 2010^{6}\end{array}$ & Caso-controle & 141 & $\begin{array}{c}\text { Questionário desenvolvido } \\
\text { pelos pesquisadores investi- } \\
\text { gando aspectos relacionados } \\
\text { ao bruxismo. }\end{array}$ & $\begin{array}{l}\text { Sialorreia noturna, uso de } \\
\text { chupeta, hábito de roer unhas } \\
\text { ou morder lábios e objetos; } \\
\text { baixa qualidade do sono. }\end{array}$ \\
\hline 2011 & $\begin{array}{c}\text { Miamoto et al. } \\
2011^{18}\end{array}$ & Caso-controle & 180 & $\begin{array}{c}\text { Relato de pais/parentes e } \\
\text { avaliação. }\end{array}$ & $\begin{array}{l}\text { Hábito de sucção e mordida } \\
\text { cruzada posterior. }\end{array}$ \\
\hline 2012 & $\begin{array}{l}\text { Montaldo et al. } \\
2012^{19}\end{array}$ & $\begin{array}{l}\text { Estudo randomi- } \\
\text { zado }\end{array}$ & 498 & Avaliação clínica. & Fumo passivo. \\
\hline 2012 & $\begin{array}{l}\text { Ferreira-Bacci Ado } \\
\text { et al. } 2012^{20}\end{array}$ & Transversal & 80 & $\begin{array}{c}\text { Relato dos pais/parentes e } \\
\text { avaliação clínica. }\end{array}$ & $\begin{array}{l}\text { Problemas emocionais, com- } \\
\text { portamentais e distúrbios do } \\
\text { sono. }\end{array}$ \\
\hline 2014 & $\begin{array}{l}\text { Sakaguchi et al. } \\
\qquad 2014^{21}\end{array}$ & Caso-controle & 1840 & Relato dos pais/parentes. & $\begin{array}{l}\text { Problemas comportamentais, } \\
\text { refluxo e baixa qualidade do } \\
\text { sono. }\end{array}$ \\
\hline
\end{tabular}

Miller et al $^{17}$ investigaram a prevalência dos distúrbios do sono em crianças com migrâneas e descreveram os diferentes comportamentos no sono ancorado em características de enxaqueca. Após analisar 118 participantes com idade entre 2 e 12 anos, concluíram que crianças com migrâneas têm alta prevalência de distúrbios do sono, incluindo dormir pouco, presença de bruxismo e ronco. Assim, as intervenções nos hábitos de sono podem melhorar os sintomas de enxaqueca e o tratamento efetivo da enxaqueca em crianças pode ter impacto positivo no sono.

O estudo de Simões-Zenari e Bitar ${ }^{6}$ investigou a relação entre ocorrência do bruxismo e fatores associados aos hábitos orais, motricidade orofacial e funções de mastigação, respiração e deglutição em 141 crianças de 4 a 6 anos de idade. Por meio de questionários aplicados aos pais para diagnóstico do bruxismo, e avaliação clínica da motricidade orofacial, obtiveram-se resultados de que as crianças com sialorreia durante o sono, com hábito de usar chupeta, morder lábios, roer unhas ou com tônus da bochecha e tipo de mordida alterados, apresentam risco aumentado para desencadear o bruxismo.
Miamoto et al. ${ }^{18}$ avaliaram, por meio de questionários aplicados aos pais e avaliação clínica para detecção de sinais de desgaste dentário, a prevalência e possíveis fatores determinantes do bruxismo do sono em 180 crianças sem deficiência cognitiva (grupo 1), com Síndrome de Down (grupo 2) e com paralisia cerebral (grupo 3). Concluiu-se que não houve diferença estatisticamente significativa entre os grupos analisados.

Montaldo et al. ${ }^{19}$ investigaram a associação entre a exposição ao fumo passivo e o bruxismo noturno em crianças $(n=498)$. Inicialmente, as crianças foram diagnosticadas com bruxismo por meio do relato dos pais e presença de sinais e sintomas. Posteriormente, aquelas com exposição ao fumo passivo foram randomizadas em dois grupos: grupo 1 crianças que não foram expostas nos últimos seis meses e grupo 2 - aquelas que foram expostas. O trabalho concluiu que aquelas crianças com maior exposição ao fumo apresentavam bruxismo noturno.

Ferreira-Bacci Ado et al. ${ }^{20}$ avaliaram o perfil comportamental de um grupo de crianças diagnosticadas com bruxismo em vigília e/ou noturno. Um total de 29 crianças 
foram incluídas no estudo e submetidas ao diagnóstico de bruxismo por meio do relato dos pais e avaliação do perfil psicológico por intermédio de duas escalas (Rutter's Child Behavior Scale-A2 e Child Stress Scale). Nesse estudo, observaram que $82,76 \%$ das crianças com a parafunção necessitavam de intervenção psicológica ou psiquiátrica.

Sakaguchi et al. ${ }^{21}$ investigaram a relação entre problemas comportamentais, distúrbios do sono como o bruxismo e refluxo gastroesofágico. Crianças com idade média de 13,3 anos ( $n=1840)$ foram divididas em dois grupos: comportamento normal e problemas comportamentais. Para a avaliação do refluxo, utilizou-se a Escala de Frequência dos Sintomas do refluxo Gastroesofágico (FSSG). Para a análise do bruxismo noturno e outras desordens relacionadas ao sono, os pais foram questionados quanto à presença dos episódios. Nesse estudo, concluíram que há correlação entre essas variáveis, influenciando no estilo de vida e hábitos alimentares.

\section{Dıscussão}

A presente revisão se propôs a avaliar a literatura existente acerca dos fatores etiológicos e fatores de risco do bruxismo na população infantil. Este estudo confirma o caráter multifatorial desse distúrbio. Fatores genéticos, sistêmicos, psicossociais, desordens do sono e o fumo passivo parecem estar associados ao bruxismo.

Acredita-se que o bruxismo possa ser desencadeado pela influência de fatores emocionais resultantes da necessidade de lidar com uma série de tarefas cotidianas, perdas, expectativas, conflitos e ansiedade. Nesse contexto, o bruxismo seria uma manifestação em resposta ao modo como os indivíduos reagem emocionalmente em situações adversas, sendo que estudos apontam sua prevalência em pessoas mais ansiosas, agressivas e hiperativas. ${ }^{11,22}$ Sakaguchi et al. ${ }^{21}$ avaliaram por meio de questionários com os pais a associação do fator psicológico com a etiologia do bruxismo. Em uma amostra de 1.840 estudantes, os indivíduos com problemas de comportamento apresentaram maior prevalência desse hábito parafuncional $(40,8 \%)$, além de sonolência durante o dia $(20,8 \%)$ e baixa qualidade do sono $(57,3 \%)$. Esses achados são semelhantes ao estudo de Ferreira-Bacci Ado et al. ${ }^{20}$ que observaram, de acordo com relato dos pais, que $82,76 \%$ das crianças diagnosticadas com bruxismo também exibiam problemas comportamentais ou emocionais. Similarmente, o trabalho de Saulue et al. ${ }^{23}$ afirma que a probabilidade do bruxismo é 3,6 maior se a criança sofre alguma desordem psicológica.

O bruxismo tem sido também associado a distúrbios do sono que comprometem a qualidade do sono da criança, como ronco, respiração bucal, sono agitado, suor e fala noturnos. ${ }^{24,25}$ Ferreira-Bacci et al. ${ }^{20}$ concluíram que $1 \%$ das crianças diagnosticadas com bruxismo do sono apresentavam outros distúrbios do sono concomitantes. Tais distúrbios afetam diretamente a qualidade de vida das crianças, por acarretarem um padrão de sono não repousante, fato que reflete no desempenho educacional e nas atitudes comportamentais da criança. ${ }^{9,2}$ No estudo de Simões-Zenari e Bitar ${ }^{6}$ observou-se que as crianças com hábitos orais como sialorreia durante o sono, uso de chupeta, morder lábios e roer unhas, apresentam risco aumentado de desenvolver bruxismo. Além do mais, verificouse que crianças com número de horas de sono reduzido, e, por conseguinte, baixa qualidade de sono, tinham risco de bruxismo aumentado em cinco vezes. Os achados de SimõesZenari e Bitar ${ }^{6}$ sustentam a hipótese de possível associação entre distúrbios do sono concomitantemente ao bruxismo. A presença da sialorreia é um indício de respiração oral 
noturna que, por sua vez, pode estar associada à obstrução nasal e presença da Síndrome da Apneia Obstrutiva do Sono, distúrbio respiratório do sono com impacto direto sobre nível de fragmentação, eficiência e qualidade de sono e quantidade de horas de sono. ${ }^{26,27}$

Os traços de personalidade de um indivíduo refletem a maneira que ele lida com seus conflitos diários e, portanto, sendo o bruxismo um mecanismo de liberação de tensões, está diretamente relacionado a determinados traços de personalidade da infância que, inclusive, podem se estender à vida adulta. O neuroticismo e a responsabilidade são traços de personalidade que se caracterizam, respectivamente, pela impaciência e autodisciplina. Por poderem estar associados a reações de estresse, ansiedade e pânico, têm sido vinculados ao desenvolvimento do bruxismo. ${ }^{12,22}$ Ferreira-Bacci Ado et al. ${ }^{20}$ analisaram traços de personalidade de crianças diagnosticadas com bruxismo. Considerando um total de 29 indivíduos recrutados, observouse que $0,69 \%$ eram crianças tímidas, $0,59 \%$ inseguras, $0,48 \%$ mostravam mau humor, $0,76 \%$ impacientes, $0,79 \%$ preocupadas. Além disso, concluíram que, geralmente, essas crianças têm dificuldade de expressar seus sentimentos, passando facilmente por situações de aflição.

Além do fator psicológico, tem se encontrado uma associação entre essa parafunção e alterações sistêmicas como o refluxo gastroesofágico, assim como, no estudo de Sakaguchi et al., ${ }^{21}$ sendo o refluxo um fator de risco para o bruxismo noturno. Essa correlação é ancorada no aumento da atividade rítmica dos músculos da mastigação induzido pela acidificação esofágica proveniente do refluxo gastroesofágico. ${ }^{21,28}$ Dickman et al. ${ }^{29}$ sugerem que a qualidade do sono está relacionada com a gravidade do refluxo e que a insônia ocorre nos pacientes no dia seguinte à exposição ácida originária do refluxo gastroesofágico.
Ademais, a enxaqueca também foi relatada como fator desencadeador do bruxismo e de outros distúrbios do sono. O estudo de Miller et al. ${ }^{17}$ mostrou que crianças com migrâneas possuíam bruxismo, dormiam poucas horas, dormiam com os pais ou roncavam. Esses achados vão ao encontro do estudo de Masuko et al., ${ }^{30}$ que objetivou investigar a prevalência do bruxismo durante o sono em crianças com episódio de migrâneas comparado ao grupo controle. Nesse trabalho, $25 \%$ das crianças, que apresentavam episódios de enxaqueca, exibiram bruxismo durante o sono, enquanto esses dados não foram observados no grupo controle. Tem-se sugerido que a enxaqueca e o sono apresentam um possível substrato genético comum. Estudos mostraram correlação entre enxaqueca e perturbações do sono em adultos, mas ainda não existem evidências científicas para confirmar essa associação em crianças. ${ }^{31}$

A exposição ao fumo e, em especial, à nicotina, tem sido considerada um fator de risco ao bruxismo, imaginando que a nicotina está associada a uma maior atividade muscular e ao aumento da atividade dopaminérgica. Um estudo randomizado com 498 crianças mostrou que aquelas expostas ao fumo passivo apresentaram maior risco de desenvolver o bruxismo do sono. Durante o estudo, os pais das crianças foram orientados a pararem de fumar e, após seis meses sem exposição ao fumo passivo, o número de crianças com a parafunção diminuiu significativamente. ${ }^{19} \mathrm{O}$ fumo passivo e a exposição direta ao fumo são, comprovadamente, coadjuvantes no desenvolvimento do bruxismo em adolescentes e adultos, e, assim, acredita-se que também seriam fatores de risco para o bruxismo infantil. ${ }^{8}$

Distúrbios neurológicos e, principalmente, quando associados a distúrbios cognitivos, assim como na Síndrome de Down, são considerados fatores de risco à ocorrência de distúrbios estereotipados do movimento, 
como o bruxismo secundário. ${ }^{32}$ No trabalho de Ortega et al., ${ }^{33}$ ao compararem a presença de apertamento dentário em crianças com paralisia cerebral e aquelas com ausência desse distúrbio, observou-se que a presença da parafunção é significativamente maior no primeiro grupo. Contudo, o estudo de Miamoto et al., ${ }^{18}$ que comparou a prevalência do bruxismo e de fatores associados em crianças com Síndrome de Down, com paralisia cerebral e em crianças sem deficiências cognitivas, não encontrou diferença significativa de prevalência. Isso pode ser atribuído à adoção de metodologias distintas quanto aos critérios de inclusão e critérios de diagnóstico do bruxismo. ${ }^{18}$

A relação entre má-oclusão e bruxismo ainda se mantém controversa na literatura. Estudos mais recentes confirmam a ausência de evidência científica suficiente para suportar a hipótese de que o bruxismo seja influenciado por fatores periféricos, como a presença de interferências oclusais. ${ }^{5,12}$ Miamoto et al. ${ }^{18}$ concluíram que hábitos de sucção e mordida cruzada são manifestações diretamente relacionadas ao bruxismo, sustentando a hipótese de que interferências oclusais durante movimentos da mastigação provocam uma maior tendência de desvio mandibular, favorecendo, assim, o bruxismo.

A ausência de um padrão de diagnóstico acarreta em uma extensa variedade de metodologias de diagnóstico, fato que pode interferir nos dados de prevalência do bruxismo e nos fatores etiológicos encontrados nos estudos incluídos na atual revisão. A metodologia de diagnóstico dos estudos incluídos é ancorada na presença de sons de apertamento e/ou ranger de dentes relatado por familiares por meio de questionários específicos, ou também pela detecção de sinais e sintomas por intermédio de avaliação clínica. Considerando a ampla amostra de pacientes dos estudos incluídos na revisão, o emprego de tais metodologias se torna adequado devido à sua facilidade de execução. Além do mais, seu emprego tem respaldo da Associação Americana de Desordens do Sono. ${ }^{1}$

O bruxismo, de fato, apresenta etiologia multifatorial, sendo os fatores etiológicos e de risco amplamente variáveis. Na atual revisão, a presença de problemas comportamentais e emocionais foi considerado um fator de risco ao bruxismo infantil em parte significativa dos artigos incluídos. A presença de outros distúrbios do sono que também comprometem a qualidade do sono, com reflexo na quantidade de horas de sono, também se destacou como fator de risco significante. A presença de fatores de risco, que não se limitam à área de atuação do cirurgião-dentista, reforça a necessidade de uma terapêutica multidisciplinar para o tratamento do bruxismo infantil. Porém, o conhecimento dos possíveis fatores de risco e etiológicos capacita o cirurgião-dentista a adotar uma abordagem mais completa e efetiva, evitando a progressão dos efeitos deletérios do bruxismo e das possíveis comorbidades associadas a ele.

\section{Considerações Finais}

O bruxismo infantil tem sido associado a diversos fatores etiológicos e de risco e, sendo assim, é de extrema importância que o cirurgião dentista tenha conhecimento acerca dos fatores envolvidos na fisiopatologia do bruxismo, para que uma melhor abordagem seja conduzida.

\section{REFERÊnCIAS}

1. Sateia MJ. International classification of sleep disorders-third edition: highlights and modifications. Chest., 2014, 146 (5): 1.387-1.394. https://www.sciencedirect.com/science/article/ pii/S0012369215524070?via\%3Dihub 
2. Lobbezoo F., Ahlberg J., Glaros AG, Kato T., Koyano K., Lavigne GJ, et al. Bruxism defined and graded: an international consensus. J Oral Rehabil., 2013, 40 (1): 2-4.

https://www.ncbi.nIm.nih.gov/ pubmed/23121262

3. Serra-Negra JM, Paiva SM, Seabra AP, Dorella C., Lemos BF, Pordeus IA. Prevalence of sleep bruxism in a group of Brazilian schoolchildren. Eur Arch Paediatr Dent., 2010, 11 (4): 192-195. https://www.ncbi.nlm.nih.gov/pmc/articles/ PMC4347411/

4. Manfredini D., Restrepo C., Diaz-Serrano K., Winocur E., Lobbezoo F. Prevalence of sleep bruxism in children: a systematic review of the literature. J Oral Rehab., 2013, 40 (8): 631-642. https://onlinelibrary.wiley.com/doi/abs/10.1111/ joor.12069

5. Carra MC, Huynh N., Fleury B., Lavigne G. Overview on Sleep Bruxism for Sleep Medicine Clinicians. Sleep Med Clin., 2015, 10 (3): 375-384. https://app.dimensions.ai/details/publication/ pub.1022162289

6. Simões-Zenari M., Bitar ML. Factors associated to bruxism in children from 4-6 years. Pro Fono., 2010, 22 (4): 465-472.

http://www.producao.usp.br/bitstream/handle/ BDPI/9037/art_SIMOES-ZENARI_Fatores_ associados_ao_bruxismo_em_criancas_ de_2010.pdf?sequence $=1$

7. Ferreira NM, Santos JF, dos Santos MB, Marchini L. Sleep bruxism associated with obstructive sleep apnea syndrome in children. Cranio., 2015; 33 (4): 251-255.

https://doi.org/10.1179/215109031 4Y.0000000025

8. Castroflorio T., Bargellini A., Rossini G., Cugliari G., Rainoldi A., Deregibus A. Risk factors related to sleep bruxism in children: A systematic literature review. Arch Oral Biol., 2015, 60 (11): 1.618-1.624.

https://www.sciencedirect.com/science/article/ pii/S0003996917303576

9. Serra-Negra JM, Paiva SM, Fulgêncio LB, Chavez BA, Lage CF, Pordeus IA. Environmental factors, sleep duration, and sleep bruxism in Brazilian schoolchildren: a case-control study.

Sleep Med., 2014, 15 (2): 236-239.

https://www.ncbi.nlm.nih.gov/pubmed/24398188
10. Klasser GD, Rei N., Lavigne GJ. Sleep bruxism etiology: the evolution of a changing paradigm. J Can Dent Assoc., 2015, 81: f2.

https://www.ncbi.nIm.nih.gov/pub$\mathrm{med} / 25633110$

11. Serra-Negra JM, Paiva SM, Abreu MH, Flores-Mendoza CE, Pordeus IA. Relationship between tasks performed, personality traits, and sleep bruxism in Brazilian school children--a population-based cross-sectional study. PLoS One., 2013, 8 (11): 1-6. https://www.ncbi.nlm. nih.gov/pubmed/24244614

12. Drumond CL, Souza DS, Serra-Negra JM, Marques LS, Ramos-Jorge ML, Ramos-Jorge $J$. Respiratory disorders and the prevalence of sleep bruxism among schoolchildren aged 8 to 11 years. Sleep Breath., 2017, 21 (1): 203-208. https://www.ncbi.nIm.nih.gov/pub$\mathrm{med} / 28155103$

13. Maluly M., Andersen ML, Dal-Fabbro C., Garbuio S., Bittencourt L., de Siqueira JT, et al. Polysomnographic study of the prevalence of sleep bruxism in a population sample. J Dent Res., 2013, 92 (7): 97S-103S.

h t t p s : / / w w w. n c bi.n I m.nih.gov/ pubmed/23690359

14. Carra MC, Huynh N., Lavigne G. Sleep bruxism: a comprehensive overview for the dental clinician interested in sleep medicine. Dent Clin North Am., 2012, 56 (2): 387-413.

h t t p s: / / w w w. n c bi.n I m.nih.gov/ pubmed/22480810

15. Huynh N., Kato T., Rompré PH, Okura K., Saber M., Lanfranchi PA, et al. Sleep bruxism is associated to micro-arousals and an increase in cardiac sympathetic activity. Sleep bruxism is associated to micro-arousals and an increase in cardiac sympathetic activity. J Sleep Res., 2006, 15 (3): 339-346.

https://www.ncbi.nlm.nih.gov/pubmed/16911037

16. Dawson A., Raphael KG, Glaros A., Axelsson S., Arima T., Ernberg M., et al. Development of a quality-assessment tool for experimental bruxism studies: Reliability and validity. J Orofac Pain., 2013, 27 (2): 111-122.

https://pdfs.semanticscholar.org/6d94/641c876 a4cc58f6c407b8ef6edd59bd29adc.pdf 
17. Miller VA, Palermo TM, Powers SW, Scher MS, Hershey AD. Migraine headaches and sleep disturbances in children. Headache., 2003, 43 (4): 362-368.

https://onlinelibrary.wiley.com/doi/pdf/10.1046/ j.1526-4610.2003.03071.x

18. Miamoto CB, Pereira LJ, Ramos-Jorge ML, Marques LS. Prevalence and predictive factors of sleep bruxism in children with and without cognitive impairment. Braz Oral Res., 2011, 25 (5): 439-445.

http://www.scielo.br/pdf/bor/v25n5/11.pdf

19. Montaldo L., Montaldo P., Caredda E., D'Arco A. Association between exposure to secondhand smoke and sleep bruxism in children: a randomised control study. Tob control., 2012, 21 (4): 392-395.

http://tobaccocontrol.bmj.com/content/21/4/392.info

20. Ferreira-Bacci Ado V., Cardoso CL, Díaz-Serrano KV. Behavioral problems and emotional stress in children with bruxism. Braz Dent J., 2012, 23 (3): 246-251.

http://www.scielo.br/pdf/bdj/v23n3/a11v23n03. pdf

21. Sakaguchi K., Yagi T., Maeda A., Nagayama K., Uehara S., Saito-Sakoguchi Y., et al. Association of problem behavior with sleep problems and gastroesophageal reflux symptoms. Pediatr Int., 2014, 56 (1): 24-30.

https://onlinelibrary.wiley.com/doi/pdf/10.1111/ ped.12201

22. Serra-Negra JM, Ramos-Jorge ML, Flores-Mendoza CE, Paiva SM, Pordeus IA. Influence of psychosocial factors on the development of sleep bruxism among children. Int J Paediatr Dent., 2009, 19 (5): 309-317.

https://onlinelibrary.wiley.com/doi/epdf/10.1111/ j.1365-263X.2009.\$00973.x

23. Saulue P., Carra MC., Laluque JF., d'Incau E. Understanding bruxism in children and adolescents. Int Orthod., 2015, 13 (4): 489-506.

https://ac.els-cdn.com/S1761722715000777/1s2.0-S1761722715000777-main.pdf? 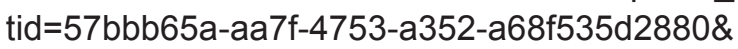 acdnat=1524770343_8fb654606439498d77e3 d866339be041
24. Guo H., Wang T., Li X., Ma Q., Niu X., Qiu J. What sleep behaviors are associated with bruxism in children? A systematic review and meta-analysis. Sleep Breath., 2017, 21 (4): 1.013-1.023.

https://link.springer.com/article/10.1007\%2 Fs11325-017-1496-3

25. Serra-Negra JM, Ribeiro MB, Prado IM, Paiva $\mathrm{SM}$, Pordeus IA. Association between possible sleep bruxism and sleep characteristics in children. Cranio., 2017, 35 (5): 315-320. https://doi.org/10.1080/08869634.2016.1239894

26. Ohayon MM, Li KK, Guilleminault C. Risk factors for sleep bruxism in the general population. Chest., 2001, 119 (1): 53-61. https://ac.els-cdn.com/S0012369215375644/1s 2. 0 - S $0012369215375644-\mathrm{ma}$ in . pdf?_tid=e5428f9c-68d1-484e-861f366440de7431\&acdnat $=1524770753$ _dd6f64c 649b316f5664091377b643fbd

27. Tachibana M., Kato T., Kato-Nishimura K., Matsuzawa S., Mohril I., Taniike M. Associations of sleep bruxism with age, sleep apnea, and daytime problematic behaviors in children. Oral Dis., 2016, 22 (6): 557-565.

https://doi.org/10.1111/odi.124§92

28. Mengatto CM., Dalberto Cda S., Scheeren B., Barros SG. Association between sleep bruxism and gastroesophageal reflux disease. J Prosthet Dent. 2013, 110 (5): 349-55.

https://www.sciencedirect.com/science/article/ pii/S0022391313000991?via\%3Dihub

29. Dickman R., Green C., Fass SS, Quan SF, Dekel R., Risner-Adler S., et al. Relationships between sleep quality and $\mathrm{pH}$ monitoring findings in persons with gastroesophageal reflux disease. J. Clin. Sleep Med., 2007, 3 (5): 505-513. https://www.ncbi.nIm.nih.gov/pmc/articles/ PMC1978337/

30. Masuko AH., Villa TR., Pradella-Hallinan M., Moszczynski AJ., Carvalho Dde S., Tufik S., et al. Prevalence of bruxism in children with episodic migraine--a case-control study with polysomnography. BMC Res Notes., 2014, 14 (7): 298. http://www.oralhealth.ro/volumes/2014/volume-4/Paper699.pdf

31. De Luca Canto G., Singh V., Bigal ME, Major PW, Flores-Mir C. Association between tensiontype headache and migraine with sleep brux- 
ism: a systematic review. Headache. 2014, 54 (9): 1.460-1.469.

https://www.ncbi.nlm.nih.gov/pubmed/25231339

32. Ella B., Ghorayeb I., Burbaud P., Guehl D. Bruxism in Movement Disorders: A Comprehensive Review. J Prosthodont., 2016, 26 (7): 599-605. https://www.ncbi.nIm.nih.gov/pub$\operatorname{med} / 27077925$
33. Ortega AO., Dos Santos MT., Mendes FM., Ciamponi AL. Association between anticonvulsant drugs and teeth-grinding in children and adolescents with cerebral palsy. J Oral Rehabil., 2014, 41 (9): 653-658.

https://onlinelibrary.wiley.com/doi/full/10.1111/ joor.12185

Submetido em: 4-9-2017

Aceito em: 22-4-2018 